\title{
Burkholder Theorem in Riesz spaces
}

\author{
Youssef Azouzi \\ Research Laboratory of Algebra, Topology, Arithmetic, and Order \\ Faculty of Mathematical, Physical and Natural Sciences of Tunis \\ Tunis-El Manar University, 2092-El Manar, Tunisia \\ Kawtar Ramdane \\ Moroccan Polytechnic Research and Innovation Center (CMRPI)
}

\begin{abstract}
The main purpose of this paper is to give a vector lattice version of a Theorem by Burkholder about convergence of martingales. The proof is based on a vector lattice analogue of Austin's sample function theorem, proved recently by Grobler, Labuschagne and Marraffa and on a new characterization of elements of the sup-completion of a universally complete vector lattice which do not belong to the space.
\end{abstract}

\section{Introduction}

This paper aims to generalize the following theorem due to Burkholder in the setting of vector lattices. Our proof presented here follows Chen [7].

Theorem 1 Let $(\Omega, \mathcal{F}, P)$ be a probability space. Consider two martingales $f=\left(f_{n}\right)_{n \geq 1}$ and $g=\left(g_{n}\right)_{n \geq 1}$ with respect to the same filtration $\left(\mathcal{F}_{n}\right)_{n \geq 1}$ such that $\|f\|_{1}:=\sup \left\|f_{n}\right\|_{1}<\infty$ and $S_{n}(g) \leq S_{n}(f)$ for $n=1,2, \ldots$ Then $g$ converges almost surely.

Here $S_{n}(h)$ denotes the Quadratic Variation Process associated to $h$, that is, $S_{n}(h)=\sum_{k=1}^{n}\left(h_{k}-h_{k-1}\right)^{2}$.

In the framework of vector lattices we will exclusively consider Dedekind complete vector lattices, so every bounded nonempty subset of $E$ has a supremum and infimum. Comparing with the classical probability theory, one can 
think of the space $L_{1}=L_{1}(\Omega, \mathcal{F}, \mathbb{P})$ consisting of all integrable real random variables. The space $E^{u}$, the universal completion of $E$, plays the same role as $L_{0}$ does in the classical theory, while $E_{s}$, the sup-completion of $E$ corresponds to the cone of random variables taking their values in $\mathbb{R} \cup\{\infty\}$ which are bounded below by some integrable variable. Recall that $E^{u}$ is Dedekind complete and laterally complete, where the later means that every subset of $E^{u}$ which consists of mutually disjoint positive elements, has a supremum in $E^{u}$. However, an arbitrary subset of $E^{u}$ need not have a supremum. This lack of supremum could be made up by considering the sup-completion $E_{s}$ of $E$. This concept is due to Donner in [9] and we refer to [9, 2] for the definition and properties of the sup-completion $E_{s}$. We just recall here that $E_{s}$ is a unique Dedekind complete ordered cone that contains $E$ as a sub-cone of its group of invertible elements and in which every nonempty subset has a supremum. The role of a probability measure is played by a conditional expectation operator, i.e., a strictly positive order continuous projection $T$ mapping the vector lattice into itself onto a Dedekind complete Riesz subspace with $T e=e$ form some weak order unit $e$. A great part of this theory has been developed by Kuo, Labuschagne and Watson (see for example [19, 20, 21]) for the discrete case and essentially by Grobler, Labuschagne for the continuous case (see for example [11, 12, 15, 13, 14]). The central result of this work is to get a generalization of Theorem 1. Several nice results can be discovered in the way to reach our purpose. We believe that it is the case here for Theorem 10 and Theorem 3, which concern order structure and are totally independent of the probability theory. Both of them are crucial steps to prove our main result. The first result provides a characterization of elements in $E_{s}$ that do not belong to $E$, where $E$ is a universally complete vector lattice. It states that a positive element $x$ in $E_{s}$ belongs to $E$ if and only if $\inf _{\lambda \in(0, \infty)} P_{(x-\lambda e)^{+}} e=0$, where $P_{u}$ denotes the order projection on the band generated by $u$. This condition is the abstract translation of the fact that $\mathbb{P}(|X| \geq \lambda) \downarrow 0$ as $\lambda \longrightarrow \infty$, which means that the random variable $X$ is finite. For more informations about the connection between $E^{u}$ and $E_{s}$ we refer to [2]. The second result, Theorem [3, provides a sufficient condition for a sequence $\left(x_{n}\right)_{n>1}$ in $E$ to converge in order in the space $E^{u}$. This condition says that it is enough to prove that for some sequence of band projections $\left(P_{k}\right)$ satisfying $P_{k} \uparrow I$, the sequences $\left(P_{k} x_{n}\right)_{n \geq 1}, k=1,2, \ldots$ converges in order. In the proof of this result we use the notion of unbounded order convergence, which characterizes the notion of almost everywhere convergence 
in the classical theory, and has received increasing attention in recent years (see for example [10, 16]). In order to prove some other intermediate results we are led to use the notion of Riemann integral in vector lattices introduced by the authors in [3]. All these results are proved in Section 2. In that section we collect all results we need to prove the main theorem of this paper (Theorem 17). Its proof is presented in Section 3.

As we have pointed out above, we will consider a Dedekind complete vector lattice $E$ and we assume that $E$ has a weak order unit $e$. This space will be equipped with a conditional expectation operator $T$ such that $T e=e$. This distinguished unit $e$ plays the same role as the constant random variable 1 does in the classical theory..It was shown in [19] that there exists a largest Riesz subspace of $E^{u}$, called the natural domain of $T$, and denoted by $L^{1}(T)$, to which $T$ extends uniquely to a conditional expectation which will denoted again by $T$. It is worth noting that $L^{1}(T)$ is a Dedekind complete ideal of $E^{u}$. It is also $T$-universally complete, that is, for every increasing net $\left(x_{\alpha}\right)$ in $L^{1}(T)$ such that $\left(T x_{\alpha}\right)_{\alpha}$ is bounded in $E^{u}$, the supremum $\sup x_{\alpha}$ exists in $L^{1}(T)$. In [12] Grobler introduced the notion of Daniell Integral in vector lattices and used it to develop a kind of functional calculus. These tools has been considered to study the spaces $\mathcal{L}^{p}(T)$ for $p \in[1, \infty)$ in 4 ] by the first author and Trabelsi. For $p \in[1, \infty)$ the space $L^{p}(T)$ is equipped with the a vector valued norm \|\|$_{p, T}$ defined by

$$
\|f\|_{p, T}=\left(T|f|^{p}\right)^{1 / p}, \quad f \in L^{p}(T) .
$$

Notions of filtrations, martingales have been defined in the measure-free framework of vector lattices by means of operators. All of them are needed to give an abstract formulation of Burkholder Theorem. Let us recall some definitions. A filtration in $E$ is a sequence of conditional expectation operators $\left\{T_{n}: n \geq 1\right\}$ with $T_{1}=T$ and $T_{i} T_{j}=T_{j} T_{i}=T_{\min (i, j)}$ for all $i, j \geq 1$. ([20, Definition 3.1]). A martingale is a sequence $\left(x_{n}\right)_{n \geq 1}$ of elements of $E$ such that $T_{n} x_{n+1}=x_{n}$ for all $n \geq 1$. Consider now two martingales $\left(f_{n}\right)_{n \geq 1}$, and $\left(g_{n}\right)_{n \geq 1}$ and define $\|f\|_{1}=\sup _{n} T\left|f_{n}\right| \in E_{s}$. Keeping the same notations as in the concrete case, it turns out that $g$ converges in order in $E^{u}$ whenever $S_{n}(g) \leq S_{n}(f)$ for $n=1,2, \ldots$ and $\|f\|_{1}$ exists in $E^{u}$. For the proof of this result we need also the notions of stopping time and stopped process in the setting of Riesz spaces. We recall from [20] that a stopping time is an increasing sequence $\left(P_{i}\right)_{i \geq 1}$ of band projections on $E$ such that

$$
P_{i} T_{j}=T_{j} P_{i} \text { whenever } 1 \leq i \leq j .
$$


Given a filtration $\left(T_{n}\right)$, an adapted process $f=\left(f_{n}\right)$, that is, $\left(f_{n}\right)$ is a sequence in $E$ with $f_{n} \in R\left(T_{n}\right)$, for all $n \in \mathbb{N}$, and a stopping time $P=\left(P_{n}\right)$, we can define the stopped process $\left(f_{p}, T_{p}\right)$ by putting

$$
f_{P}=\sum_{i=1}^{\infty}\left(P_{i}-P_{i-1}\right) f_{i} .
$$

One of the key ideas in the proof of Burkholder Theorem is a vector lattice version of Austin Theorem, which is the main result of [15]. For more informations about Riesz spaces we refer to [1, 24] and for probability theory we refer to [23] and [8].

\section{Several tools}

This section contains all results needed to prove our main Theorem (Theorem 17). We start be giving a brief review of unbounded order convergence. This kind of convergence is a satisfactory abstraction of almost everywhere convergence A net $\left(x_{\alpha}\right)_{\alpha \in A}$ in a vector lattice $E$ is said to be order convergent to $x$, and we write $x_{\alpha} \stackrel{o}{\longrightarrow} x$, if there exists a net $\left(y_{\beta}\right)_{\beta \in B}$ such that $y_{\beta} \downarrow 0$ and for each $\beta \in B$ there exists $\alpha_{\beta} \in A$ satisfying $\left|x_{\alpha}-x\right| \leq y_{\beta}$ for all $\alpha \geq \alpha_{\beta}$. A net $\left(x_{\alpha}\right)$ is said to converge in unbounded order to $x$, and we write

$x_{\alpha} \stackrel{u o}{\longrightarrow} x$, if $\left|x_{\alpha}-x\right| \wedge y \stackrel{o}{\longrightarrow} 0$ for every $y \in E_{+}$. If $E$ has a weak unit $e$ this can be reduced to $\left|x_{\alpha}-x\right| \wedge e \stackrel{o}{\longrightarrow} 0$. (see Theorem 2.2. in [18]) It should be noted that these convergences agree for sequences if the space is universally complete [10, Corollary 3.12]. For more informations about uo-convergence the reader is referred to papers [18] and [10]. It is sometimes convenient to use series instead of sequences. The notation $\sum_{k=1}^{\infty} x_{k}$ means the order limit of the sequence $S_{n}=\sum_{k=1}^{n} x_{k}$. Our first important result in this section provides a sufficient condition for a sequence $\left(x_{n}\right)$ in $E$ to be order convergent in $E^{u}$. Let us record a lemma which will be of great use for us; see e.g., [24, Corollary $32.4]$.

Lemma 2 Let $E$ be a vector lattice. If the projection bands $B_{1}, B_{2}, B_{3}, B_{4}$ in $E$ with corresponding band projections $P_{1}, P_{2}, P_{3}, P_{4}$ satisfy $B_{4} \subset B_{3} \subset$ $B_{2} \subset B_{1}$ then $\left(P_{1}-P_{2}\right) \perp\left(P_{3}-P_{4}\right)$ and so $\left(P_{1}-P_{2}\right) u \wedge\left(P_{3}-P_{4}\right) u=0$ for every $u \in E^{+}$. 
Theorem 3 Let $E$ be a Dedekind complete vector lattice and consider a sequence of band projections $\left(P_{k}\right)_{k \geq 1}$ such that $P_{k} \uparrow I$ and a sequence $\left(x_{n}\right)_{n \geq 1}$ of elements of $E$. If for each $k$ the sequence $\left(P_{k} x_{n}\right)_{n \geq 1}$ converges in order in $E$, then $\left(x_{n}\right)_{n \geq 1}$ converges in order in $E^{u}$.

Proof. By considering the positive part and the negative part of $x_{n}$ we may assume, without loss of generalities, that $x_{n} \in E^{+}$for all $n$. We denote by $y_{k}$ the order limit of $\left(P_{k}\left(x_{n}\right)\right)_{n \geq 1}$. The sequence $\left(y_{k}\right)_{k \geq 1}$ is increasing because $\left(P_{k}\right)_{k \geq 1}$ is so. Moreover, we have

$$
P_{k-1} y_{k}=\lim _{n} P_{k-1} P_{k} x_{n}=y_{k-1} .
$$

Since we have also $y_{k}=P_{k} y_{k}$ we obtain

$$
y_{k}=\sum_{i=1}^{k}\left(P_{i}-P_{i-1}\right) y_{i},
$$

where $P_{0}=0$. Now since $\left(P_{i}-P_{i-1}\right)$ is a sequence of disjoint projections we see that

$$
y=\sup y_{k}=\sum_{i=1}^{\infty}\left(P_{i}-P_{i-1}\right) y_{i}
$$

exists in $E^{u}$. We claim now that $x_{n} \stackrel{o}{\longrightarrow} y$ in $E^{u}$. As it was noted above, it is sufficient to prove that $\left(x_{n}\right)_{n \geq 1}$ converges to $y$ in unbounded order. To this end let $z \in E_{+}$and observe that

$$
\begin{aligned}
\left|x_{n}-y\right| \wedge z & =P_{k}\left(\left|x_{n}-y\right| \wedge z\right)+P_{k}^{d}\left(\left|x_{n}-x\right| \wedge z\right) \\
& \leq P_{k}\left(\left|x_{n}-y\right| \wedge z\right)+P_{k}^{d} z
\end{aligned}
$$

which yields

$$
\limsup _{n \longrightarrow \infty}\left|x_{n}-y\right| \wedge z \leq P_{k}\left|y_{x}-y\right|+P_{k}^{d} z \leq\left|y_{k}-y\right|+P_{k}^{d} z .
$$

and the result follows since $P_{k}^{d} z \stackrel{o}{\longrightarrow} 0$ and $\left|y-y_{k}\right| \stackrel{o}{\longrightarrow} 0$ as $k \longrightarrow \infty$.

Remark 4 Does the above theorem remain valid for nets? As order convergence and unbounded order convergence do not agree for nets even in universally complete vector lattices (see [22, Example 6]), it seems more reasonable to check for a counterexample. However, a slight modification of the above 
proof yields that if we take a net $\left(x_{a}\right)$ instead of a sequence in Theorem 3 , we can conclude that $\left(x_{\alpha}\right)$ is uo-convergent in $E^{u}$ and its uo-limit $y$ satisfies $y=\lim y_{k}=\sup y_{k}$, where $y_{k}$ is the order limit of $\left(P_{k} x_{\alpha}\right)_{\alpha \in A}$. Now if we look for a more general result we will take a net of band projections. In this case the construction of the uo-limit of $\left(x_{\alpha}\right)$ is less obvious, but the result remains true as will be shown in the next result.

Theorem 5 Let $E$ be a Dedekind complete vector lattice and consider a net of band projections $\left(P_{\gamma}\right)_{\gamma \in \Gamma}$ such that $P_{\gamma} \uparrow I$ and a net $\left(x_{\alpha}\right)_{\alpha \in A}$ of elements in $E$. If for each $\gamma$ the net $\left(P_{\gamma} x_{\alpha}\right)_{\alpha \in A}$ is order convergent, then $\left(x_{\alpha}\right)$ is uoconvergent in $E^{u}$.

Proof. We denote $y_{\alpha}=\lim _{\gamma} P_{\gamma} x_{\alpha}$ for each $\alpha \in A$. As in the proof of Theorem 3 we can show that $\left(y_{\alpha}\right)_{\alpha \in A}$ is an increasing net, but it is not obvious how to show that its supremum is still in $E^{u}$. We will prove instead that the net $\left(x_{\alpha}\right)$ is uo-Cauchy and conclude then by [2, Theorem 17]. To this end let $y \in E_{+}$and observe that

$$
\begin{aligned}
\left|x_{\alpha}-x_{\beta}\right| \wedge y & =P_{\gamma}\left|x_{\alpha}-x_{\beta}\right| \wedge y+P_{\gamma}^{d}\left|x_{\alpha}-x_{\beta}\right| \wedge y \\
& \leq P_{\gamma}\left(\left|x_{\alpha}-y_{\gamma}\right|+P_{\gamma}\left|x_{\beta}-y_{\gamma}\right|\right)+P_{\gamma}^{d} y
\end{aligned}
$$

It follows that limsup $\left|x_{\alpha}-x_{\beta}\right| \wedge y \leq P_{\gamma}^{d} y$ for all $\gamma \in \Gamma$ and then lim sup $\left|x_{\alpha}-x_{\beta}\right| \wedge$

$$
(\alpha, \beta) \quad(\alpha, \beta)
$$

$y=0$ which proves that $\left(x_{\alpha}\right)$ is uo-Cauchy as required.

As it was mentioned in Remark 4, the order convergence can not be expected in the above Theorem.

Example 6 Let $E=\ell_{\infty}$ and consider the net $\left(x_{\alpha}\right)_{\alpha \in A}$ defined by: $x_{(p, q)}=$ $q \sum_{p \leq k \leq q} e_{k}$, where the indexed set, $A=\{(p, q) \in \mathbb{N} \times \mathbb{N}: p \leq q\}$, is ordered cordinatwise and $\left(e_{k}\right)$ is the standard basis of $\ell_{\infty}$. Let $P_{k}$ be the band projection on the principal band generated by $e_{1}+\ldots+e_{k}$. It is easy to check that $P_{k} x_{\alpha} \stackrel{o}{\longrightarrow} 0$ for every $k$. However, $\left(x_{\alpha}\right)$ is not order convergent in $\ell_{\infty}^{u}$ because it hasn't an order bounded tail. Indeed if $\alpha_{0}=\left(p_{0}, q_{0}\right)$ is fixed then for $q>q_{0} \geq p_{0}$ and $\alpha_{q}=\left(p_{0}, q\right)$ we have $\alpha_{q} \geq \alpha_{0}$ and $x_{\alpha_{q}} \geq q e_{p_{0}}$.

It is frequent in classical probability theory to consider random variables with values in the extended real line, and most often in $\mathbb{R}_{\infty}=\mathbb{R} \cup\{\infty\}$, 
and then it is question to check if these variables are finite almost everywhere. The corresponding situation in our model of measure-free theory, random variables finite almost everywhere correspond to elements of $E^{u}$, and random variables with values in $\mathbb{R}_{\infty}$ correspond to elements of $E_{s}$, the sup-completion of $E$ and it was a challenge for us to recognize 'finite elements' of $E_{s}$, those that belong to $E^{u}$. In [2, Theorem 14], the first author obtains a characterization of elements of $E_{s}$ which do not belong to $E^{u}$ for a Dedekind complete vector lattice $E$; that characterization is a crucial step in the proof of the main theorem in [2], which states that a vector lattice is uo-complete if and only if it is universally complete, so to prove that a net in $E^{u}$ is uo-convergent it is sufficient to show that it is uo-Cauchy, as it has been done in the proof of Theorem 5. As the proof of [2, Theorem 14] is involved and will be only applied in the case of universally complete spaces, we provide here an alternative proof of this result in that special case, which is less involved. Let us first recall some notations and facts. Consider a Dedekind complete vector lattice $E$ with a weak order unit $e$ and denote by $E_{s}$ its sup-completion. If $a \in E^{+}$, we denote by $P_{a}$ the band projection on the principal band generated by $a$. It satisfies:

$$
P_{a}(x)=\sup _{k}(x \wedge k a), \quad \text { for all } x \in E^{+} .
$$

We shall use this formula to extend $P_{a}$ for all positive elements $x$ in $E_{s}$. On the other hand if $a$ is a positive element in $E_{s}$, we can define the map $\varphi_{a}$ on $E$ by putting

$$
\varphi_{a}(x)=\sup _{n \in \mathbb{N}}(x \wedge n a)=\sup _{\lambda>0}(x \wedge \lambda a)
$$

for all $x \in E_{+}$and then $\varphi_{a}(x)=\varphi_{a}\left(x^{+}\right)-\varphi_{a}\left(x^{-}\right)$for all $x \in E$. The next lemma shows that $\varphi_{a}$ is a band projection on $E$.

Lemma 7 [2, Lemma 4]Let $E$ be a Dedekind complete vector lattice with a weak order unit e. If $a \in E_{s}^{+}$, then $\varphi_{a}=P_{\varphi_{a}(e)}$.

Remark 8 It is easily checked that $P_{a}(x)=\sup _{\beta} P_{a}\left(x_{\beta}\right)$ for each net $\left(x_{\beta}\right)$ in $E_{+}$which increases to $x \in E_{s}^{+}$. Instead of $\varphi_{a}$ we prefer the notation $P_{a}$ even if a in a positive element in $E_{s}$.

The following lemma will be needed in the proof of the second main result of this section. 
Lemma 9 Let $E$ be a Dedekind complete vector lattice with weak order unit and let $P$ and $Q$ be two disjoint band projections. Then

$$
(P+Q) z=(P \vee Q) z \quad \text { for all } z \in E_{s}^{+} .
$$

Proof. Let $\left(z_{\alpha}\right)_{\alpha \in \Gamma}$ be a net in $E^{+}$such that $z_{\alpha} \uparrow z$. We have seen that $R z=$ $\sup R z_{\alpha}$ for all band projection $R$. Since $(P \vee Q) z_{\alpha}=P z_{\alpha}+Q z_{\alpha} \leq P z+Q z$ holds for all $\alpha$ we get the inequality

$$
(P \vee Q) z \leq P z+Q z
$$

For the reverse inequality we need the following fact: If $A, B$ are two nonempty subsets of $E$ then $\sup (A+B)=\sup A+\sup B$ in $E_{s}$ (see the proof of [9, Theorem 1.4]). Apply this to subsets $A=\left\{P z_{\alpha}\right\}$ and $B=\left\{Q z_{\alpha}\right\}$ to obtain

$$
P z+Q z=\sup _{\alpha, \beta}\left(P z_{\alpha}+Q z_{\beta}\right) .
$$

Now, given $\alpha, \beta$ in $\Gamma$, there exists $\gamma \in \Gamma$ such that $\gamma \geq \alpha$ and $\gamma \geq \beta$, hence

$$
P z_{\alpha}+Q z_{\beta} \leq P z_{\gamma}+Q z_{\gamma}=(P \vee Q) z_{\gamma} \leq(P \vee Q) z
$$

Taking the supremum over $\alpha, \beta$ we get

$$
(P+Q) z \leq(P \vee Q) z,
$$

which yields the desired result.

Theorem 10 Let E be a universally complete vector lattice with a weak order unit $e$ and let $x \in E_{s}$, the sup-completion of $E$. Then $x \in E$ if and only if $\inf _{k>0} P_{(x-k e)}+e=0$.

Proof. The forward direction is obvious. For the converse direction assume that $\inf _{k} P_{(x-k e)}+e=0$. We will prove that $x \leq y$ for some $y \in E$, which shows that $x \in E$ because $E$ is a tight imbedding cone in $E_{s}$ (see [9]). Define two sequence of band projections by putting for $k=1,2, \ldots$,

$$
P_{k}=P_{(x-k e)^{+}}^{d}=P_{e-P_{(x-k e)^{+}}}, \quad Q_{k}=P_{k}-P_{k-1},
$$

with $P_{0}=0$. Then $Q_{i} y \wedge Q_{j} y=0$ for all $i, j \in \mathbb{N}$ with $i \neq j$, and $y \in E_{+}$by Lemma 9, and $P_{k} \uparrow I$ by our assumption. It follows therefore that

$$
x \wedge l e=\sup _{n} P_{n}(x \wedge l e)=\sup _{n} \sum_{k=1}^{n} Q_{k}(x \wedge l e)=\sum_{k=1}^{\infty} Q_{k}(x \wedge l e) .
$$


According to Lemma 9 we have that

$$
Q_{m}(z)+Q_{n}(z)=Q_{m} \vee Q_{n}(z) \text {, for all } z \in E_{s}^{+} .
$$

It follows that

$$
\begin{aligned}
x & =\sup _{l}(x \wedge l e)=\sup _{l} \sup _{n} \sum_{k=1}^{n} Q_{k}(x \wedge l e) \\
& =\sup _{n} \sup _{l} \sum_{k=1}^{n} Q_{k}(x \wedge l e)=\sup _{n} \sum_{k=1}^{n} Q_{k} x \\
& =\sum_{k=1}^{\infty} Q_{k} x .
\end{aligned}
$$

Now from the inequality

$$
x \leq \sum_{k=1}^{\infty} Q_{k}(x-k e)^{+}+\sum_{k=1}^{\infty} Q_{k} k e,
$$

and the fact that $Q_{k}(x-k e)^{+}=0$ for all $k \in \mathbb{N}$, we obtain

$$
x \leq \sum_{k=1}^{\infty} k Q_{k} e
$$

But $\sum_{k=1}^{\infty} k Q_{k} e$ belongs to $E$ as a supremum of positive disjoint sequence, so $x \in E$ as required.

Remark 11 It is worth noting that the proof presented above still valid if we assume only that $E$ is $\sigma$-universally complete.

The next corollary occurs by combining Theorem 10 with Doob Maximal Inequality [12, Theorem 6.1]. Recall that a net $\left(x_{\alpha}\right)$ in $E$ is said to be $T$ bounded if $\left(T\left|x_{\alpha}\right|\right)$ is bounded in $E$.

Lemma 12 Let $E$ be a Dedekind complete vector lattice with a weak order unit e equipped with a conditional expectation $T$ with $T e=e$. If $f=\left(f_{n}, T_{n}\right)$ is a T-bounded in $E$ then $f^{*}:=\sup \left|f_{n}\right| \in E^{u}$. 
Notice here that $f^{*}$ is well defined in $\left(E^{u}\right)_{s}$ and Theorem 10 will be used to prove that $f^{*}$ is in fact in $E^{u}$.

Proof. Put $M=\sup _{n} T\left|f_{n}\right| \in M$. By Doob Maximal Inequality [12, Theorem 6.1] we get $T P_{\left(f_{n}-\lambda e\right)^{+}} e \leq \lambda^{-1} M$. Since $P_{\left(f_{n}-\lambda e\right)^{+}}+\uparrow P_{\left(f^{*}-\lambda e\right)^{+}} e$ it follows that

$$
T P_{\left(f^{*}-\lambda e\right)^{+}} e \leq \lambda^{-1} M
$$

and then $T\left(\inf _{\lambda>0} P_{\left(f^{*}-\lambda e\right)^{+}}\right)=0$. Now using the strict positivity of $T$ to get $\inf _{\lambda>0} P_{\left(f^{*}-\lambda e\right)^{+}}+e=0$, and so the result follows by applying Theorem 10,

Before stating and proving our last result in this section (Proposition 15), that will be needed to prove the main theorem of this paper, let us say a few words about Riemann integral in vector lattices. This notion had been introduced by the authors in [3]. The theory is a faithful generalization of the classical theory. From that paper we invoke the following result [3, Lemma $5]$.

Lemma 13 Let $X$ be an order complete vector lattice with a weak order unit e. Let $p \in(0, \infty)$ and $a, \varepsilon \in(0, \infty)$ with $\varepsilon<a$. Then

$$
x^{p}-\varepsilon^{p} e=\int_{\varepsilon}^{a} p t^{p-1} P_{(x-t e)^{+}} e d t \quad \text { for all } x \in X \text { with } \varepsilon e<x \leq a e .
$$

Now if $p \in[1, \infty)$, the same proof yields the following:

Lemma 14 Let $X$ be an order complete vector lattice with a weak order unit e. Let $a \in(0, \infty)$ and $p \in[1, \infty)$. Then

$$
x^{p}=\int_{0}^{a} p t^{p-1} P_{(x-t e)+} e d t \quad \text { for all } x \in X \text { with } 0 \leq x \leq a e .
$$

We recall that for if $E$ is a Dedekind complete vector lattice with a weak order unit $e$ and if $x \geq e$ then $x$ has an inverse in the $f$-algebra $E^{u}$ (see [17, Theorem 3.4]), we denote this inverse by $x^{-1}$. Since $E$ is an ideal in $E^{u}$ and $x^{-1} \leq e$ it turns out that $x^{-1}$ belongs to $E$.

Proposition 15 Let $E$ be a Dedekind complete vector lattice with a weak order unit $e$. Let $\left(x_{n}\right)$ be a sequence in $E^{+}$and $R_{n}=\sum_{i=1}^{n} x_{i}$. Then the series $\sum x_{n}\left(e+R_{n}\right)^{-2}$ converges in order and we have

$$
\sum_{n=1}^{\infty} x_{n}\left(e+R_{n}\right)^{-2} \leq e .
$$


Proof. We assume first that the sequence $\left(x_{n}\right)$ satisfies $0 \leq x_{n} \leq \lambda e$ for some $\lambda \in(0, \infty)$ and for every $n=1,2 \ldots$ By Lemma 14 we have

$$
x_{n}=R_{n}-R_{n-1}=\int_{0}^{n a}\left(P_{n, t}-P_{n-1, t}\right) e d t,
$$

where $P_{n, t}=P_{\left(R_{n}-t e\right)^{+}}$. Applying Theorem 4 in $[3]$ to get

$$
x_{n}\left(e+R_{n}\right)^{-2}=\int_{0}^{n a}\left(e+R_{n}\right)^{-2}\left(P_{n}(t)-P_{n-1}(t)\right) e d t .
$$

Now observe that

$$
\left(e+R_{n}\right)^{-2}=\frac{\left.e+R_{n}\right)^{-2}}{\left(1+t^{2}\right)}\left(t e-R_{n}\right)\left((2+t) e+R_{n}\right)+\frac{a}{(1+t)^{2}} \leq
$$

which combined with the fact that $\left(P_{n, t}-P_{n-1, t}\right)\left(t e-R_{n}\right) \leq 0$ leads to the following:

$$
\left(e+R_{n}\right)^{-2}\left(P_{n, t}-P_{n-1, t}\right) e \leq(1+t)^{-2}\left(P_{n, t}-P_{n-1, t}\right) e
$$

and then

$$
x_{n}\left(e+R_{n}\right)^{-2} \leq \int_{\varepsilon}^{n a} \frac{1}{(1+t)^{2}}\left(P_{n}(t)-P_{n-1}(t)\right) e d t .
$$

It follows that

$$
\begin{aligned}
\sum_{k=1}^{n} x_{n}\left(e+R_{n}\right)^{-2} & \leq \int_{\varepsilon}^{n a} \frac{1}{(1+t)^{2}} \sum_{k=1}^{n}\left(P_{k, t}-P_{k-1}(t)\right) e d t \\
& \leq \int_{\varepsilon}^{n a} \frac{d t}{(1+t)^{2}} e \leq e .
\end{aligned}
$$

This proves the result for bounded sequences in the ideal $I_{e}$ generated by $e$. For general case we use our first case to get

$$
\sum_{i=1}^{n}\left(x_{i} \wedge \lambda e\right)\left(e+R_{i}^{\lambda}\right)^{-2} \leq e,
$$

for all positive real $\lambda$, where $R_{i}^{\lambda}=R_{n}=\sum_{j=1}^{i}\left(x_{j} \wedge \lambda e\right)$. It follows that

$$
\sum_{k=1}^{n} x_{n}\left(e+R_{k}\right)^{-2} \leq e
$$


As this occurs for each integer $n$ we deduce that

$$
\sum_{k=1}^{\infty} x_{k}\left(e+R_{k}\right)^{-2} \leq e
$$

which completes the proof.

\section{Burkholder Theorem}

We assume throughout that $E$ is a Dedekind complete vector lattice with a weak order unit $e$ and $T$ is a conditional expectation with $T e=e$. By taking $L^{1}(T)$ we will assume also that the space $E$ is $T$-universally complete.

Consider now a filtration $\left(T_{n}\right)_{n \geq 1}$ with $T_{1}=T$. If $x=\left(x_{n}\right)_{n \geq 1}$ is an adapted process we define the quadratic variation process associated to $x$ by putting

$$
S_{k}(x)=\sum_{j=1}^{k}\left(\Delta x_{j}\right)^{2} \quad \text { for all } k \geq 1
$$

Also, we set

$$
x_{k}^{*}=\sup _{1 \leq i \leq k} x_{i} \quad \text { for all } \quad k \geq 1 .
$$

If $P=\left(P_{n}\right)_{n \geq 1}$ is a stopping time, the stopped process $x^{P}$ is defined by

$$
x_{n}^{P}=\sum_{k=1}^{n-1} \Delta P_{k} x_{k}+P_{n-1}^{d} x_{n} .
$$

We also put

$$
x_{n}^{P-1}=\sum_{k=1}^{n-1} \Delta P_{k} x_{k-1}+P_{n-1}^{d} x_{n-1} .
$$

It follows easily from the definition that

$$
S_{n}^{P}(x)-S_{n}^{P-1}(x)=\left(\left(\Delta x_{n}\right)^{2}\right)^{P}=\left(\Delta x_{n}^{P}\right)^{2},
$$

where the last equality follows from the fact that the projections $\Delta P_{1}, \ldots, \Delta P_{n-1}, P_{n-1}^{d}$ are disjoint.

The following lemma, taken from [3], is one of the classical properties of stopped martingales and will be used later. 
Lemma 16 [3, Lemma 9] Let $\left(x_{n}\right)_{n \geq 1}$ be a submartingale, and $\left(P_{k}\right)_{k \geq 1}$ be a stopping time then we have

$$
T\left(x_{P \wedge n}\right) \leq T\left(x_{n}\right) \quad \text { for all } n=1,2, \ldots
$$

We are ready now to state our main result.

Theorem 17 Let $E$ be a Dedekind complete Riesz space with weak order unit e, equipped with a conditional expectation $T$ with $T e=$ e. Let $f=$ $\left(f_{n}\right)_{n \geq 1}$ and $g=\left(g_{n}\right)_{n \geq 1}$ be two martingales in $L^{2}(T)$ with respect to the same filtrartion $\left(T_{n}\right)_{n \geq 1}$, with quadratic variation processes $\left(S_{n}(f)\right)_{n \geq 1}$ and $\left(S_{n}(g)\right)_{n \geq 1}$ respectively. Assume that $S_{n}(g) \leq S_{n}(f)$ for all $n=1,2, \ldots$, and $N_{1}(f):=\sup _{n} T\left|f_{n}\right| \in E^{u}$. Then $\left(g_{n}\right)_{n \geq 1}$ is order convergent in $E^{u}$.

The proof of this theorem will be divided in several steps. Before starting our proof let us introduce some additional notations. Fix a positive real $\lambda$ and let $P_{n}$ be the band projection the principal band generated by

$$
a_{n}=\left(f_{n}-\lambda e\right)^{+} \vee\left(S_{n}(f)-\lambda e\right)^{+} .
$$

As $a_{n} \in R\left(T_{n}\right)$ for every $n$, the sequence $P=\left(P_{n}\right)_{n \geq 1}$ is a stopping time. Our first step to proving Theorem 17 is the following:

Lemma 18 Under the hypothesis of Theorem 17 and using the notations above, the sequence $\left(\Delta g_{n}^{P}\right)_{n \geq 1}$ is T-bounded in $E$, that is, there exists $M \in E^{+}$ such that $\sup T\left|\Delta g_{n}^{P}\right| \leq M$.

Proof. Since $P_{k} \geq P_{\left(S_{k}(f)-\lambda e\right)^{+}}$we have $P_{k}^{d}=P_{k}^{d} P_{\left(S_{k}(f)-\lambda e\right)^{+}}^{d}$, whence

$$
P_{k}^{d} S_{k}^{2}(f)=P_{k}^{d} P_{\left(S_{k}-\lambda e\right)^{+}}^{d} S_{k}^{2}(f) \leq P_{k}^{d} \lambda^{2} e
$$

This implies that

$$
\begin{aligned}
\left(S_{n}^{P-1}(f)\right)^{2} & =\sum_{k=1}^{n-1} P_{i} P_{i-1}^{d} S_{i-1}^{2}(f)+P_{n-1}^{d} S_{n-1}^{2}(f) \\
& \leq \sum_{k=1}^{n-1} P_{i} P_{i-1}^{d} \lambda^{2} e+\lambda^{2} P_{n-1}^{d} e
\end{aligned}
$$


and so we get

$$
\left(S_{n}^{P-1}(f)\right)^{2} \leq \lambda^{2} e .
$$

In a similar manner one can prove

$$
\left|f_{n}^{P-1}\right| \leq \lambda e .
$$

Moreover, we have

$$
\left(\Delta g_{n}^{P}\right)^{2} \leq\left(S_{n}^{P} g\right)^{2} \leq\left(S_{n}^{P} f\right)^{2}=P_{n}^{d}\left(S_{n}(f)\right)^{2}+P_{n}\left(S_{n}^{P}(f)\right)^{2} .
$$

Now by (21), $P_{n}^{d}\left(S_{n}(f)\right)^{2} \leq \lambda^{2} P_{n}^{d} e$ and by combining (11) and (3) we obtain

$$
\begin{aligned}
P_{n}\left(S_{n}^{P}(f)\right)^{2} & =P_{n}\left(S_{n}^{P-1}(f)^{2}\right)+P_{n}\left(\Delta f_{n}^{P}\right)^{2} \\
& \leq \lambda^{2} P_{n} e+P_{n}\left(\Delta f_{n}^{P}\right)^{2} .
\end{aligned}
$$

Using these inequalities, (4) yields

$$
\left(\Delta g_{n}^{P}\right)^{2} \leq \lambda^{2} e+P_{n}\left(\Delta f_{n}^{P}\right)^{2} \leq\left(P_{n}\left|\Delta f_{n}^{P}\right|+\lambda e\right)^{2} .
$$

Thus,

$$
\begin{aligned}
\left|\Delta g_{n}^{P}\right| & \leq P_{n}\left|\Delta f_{n}^{P}\right|+\lambda e \leq P_{n}\left|f_{n}^{P}\right|+P_{n}\left|f_{n}^{P-1}\right|+\lambda e \\
& \leq P_{n}\left|f_{n}^{P}\right|+2 \lambda e \leq \sup \sum_{i=1}^{n} \Delta P_{i}\left|f_{i}\right|+2 \lambda e .
\end{aligned}
$$

Observe that $\sup \sum_{i=1}^{n} \Delta P_{i}\left|f_{i}\right|$ exists in $E^{u}$ as the sequence $\left(\Delta P_{i}\left|f_{i}\right|\right)_{i \in \mathbb{N}}$ is disjoint. We need only show that this supremum belongs to $E$, or, in other words, that the increasing sequence $\left(\sum_{i=1}^{n} \Delta P_{i}\left|f_{i}\right|\right)_{n \geq 1}$ converges in order in $E$. Since the space $E$ is $T$-universally complete it will be enough to prove that $\sup _{n} T \sum_{i=1}^{n} \Delta P_{i}\left|f_{i}\right|$ exists in $E^{u}$. To this end applying Lemma 16 to the submartingale $\left|f_{n}\right|$ to obtain,

$$
\sup _{n} T \sum_{i=1}^{n} \Delta P_{i}\left|f_{i}\right| \leq \sup _{n} T\left|f_{n}^{P}\right| \leq \sup _{n} T\left|f_{n}\right| \leq N_{1}(f) .
$$


which proves that the sequence $\left(\sup \Delta g_{n}^{P}\right)_{n \geq 1}$ is $T$-bounded in $E$ as required. Moreover we have

$$
T\left|\sup \Delta g_{n}^{P}\right| \leq 2 \lambda e+N_{1}(f) \text {. }
$$

and the proof is finished.

Define $U_{0}=e$ and for $n \geq 1$,

$$
U_{n}=e+S_{n \wedge P}^{2}(g),
$$

We know by [17, Theorem 3.4] that $U_{n}$ is invertible in the $f$-algebra $E^{u}$ and that $U_{n}^{-1} \in E$, as $E$ is an ideal in $E^{u}$ and $U_{n}^{-1} \leq e$.

Lemma 19 With the notation as above, the series $\sum U_{n}^{-1} \Delta g_{n}^{P}$ is order convergent in $E$.

Proof. Put $A_{n}=U_{n}^{-1} \Delta g_{n}^{P}$ and $h_{n}=\sum_{i=1}^{n}\left(I-T_{i-1}\right) A_{i}$ and observe that $h_{n}$ belongs to $R\left(T_{n}\right)$ and that $T_{n-1} \Delta h_{n}=T_{n-1}\left(A_{n}-T_{n-1} A_{n}\right)=0$. This shows that $\left(h_{n}\right)_{n \geq 1}$ is a martingale. Since $T_{n-1}$ is an averaging operator we have

$$
\begin{aligned}
T_{n-1}\left(\Delta h_{n}\right)^{2} & =T_{n-1}\left(A_{n}\right)^{2}+\left(T_{n-1} A_{n}\right)^{2}-2 A_{n} T_{n-1}\left(A_{n}\right) \\
& =T_{n-1}\left(A_{n}{ }^{2}\right)-T_{n-1}\left(A_{n} \cdot T_{n-1} A_{n}\right) \leq T_{n-1}\left(A_{n}{ }^{2}\right) .
\end{aligned}
$$

It follows that

$$
\begin{aligned}
\sum_{i=1}^{n} T\left(\Delta h_{n}\right)^{2} & =\sum_{i=1}^{n} T T_{i-1}\left(\Delta h_{n}\right)^{2} \leq \sum_{i=1}^{n} T T_{i-1}\left(A_{n}{ }^{2}\right) \\
& =\sum_{i=1}^{n} T\left(A_{n}\right)^{2} .
\end{aligned}
$$

Apply Lemma [15 to the sequence $x_{n}=\left(\Delta g_{n}^{P}\right)^{2}$ to prove that $\left(h_{n}\right)_{n \geq 1}$ is bounded in $L^{2}(T)$ and then in $E$ ([4, Theorem 3.2]). Using [21, Theorem 3.5] we derive that $\left(h_{n}\right)_{n>1}$ is order convergent. Now, to prove the lemma it will be sufficient to show that the series $\sum T_{i-1} A_{i}$ is order convergent. Since

$$
T_{i-1} U_{i-1}^{-1} \Delta g_{i}^{P}=U_{i-1}^{-1} T_{i-1} \Delta g_{i}^{P}=0,
$$

we have

$$
\begin{aligned}
T \sum_{i=1}^{n}\left|T_{i-1} A_{i}\right| & =T \sum^{n}\left|T_{i-1}\left(U_{i}^{-1}-U_{i-1}^{-1}\right) \Delta g_{i}^{P}\right| \\
& \leq T \sum_{i=1}^{n} T_{i-1} \sup _{k}\left|\Delta g_{k}^{P}\right|\left(U_{i-1}^{-1}-U_{i}^{-1}\right) \\
& =T \sup _{k}\left|\Delta g_{k}^{P}\right|\left(e-U_{n}^{-1}\right) \leq T \sup _{k}\left|\Delta g_{k}^{P}\right|
\end{aligned}
$$


By Lemma 18 the sequence $T \sum_{i=1}^{n}\left|T_{i-1} A_{i}\right|$ is bounded in $E^{u}$. As $E$ is $T$ universally complete we deduce that the sequence $\sum_{i=1}^{n}\left|T_{i-1} A_{i}\right|$ is order convergent in $E$, which proves the lemma.

Proof of the main Theorem. As $f$ is a strongly bounded martingale in $E^{u}$. Austin lemma [15, Section 4] yields that $S:=\sup S_{n}(f)$ exists in $E^{u}$. We have already shown that the series $\sum\left|U_{n}^{-1} \Delta g_{n}^{P}\right|$ is order convergent. Now since the sequence $\left(U_{n}^{-1}\right)_{n \geq 1}$ is increasing and bounded it follows that the series $\sum \Delta g_{n}^{P}$ is order convergent, which implies the convergence of the sequence $\left(g_{n}^{P}\right)_{n \geq 1}$. Our purpose now is to prove the convergence of the sequence $\left(g_{n}\right)$. Notice that we have worked up to now with the fixed stopping time $P=\left(P_{\left(\left(\left|f_{n}^{*}\right|-\lambda e\right)^{+} \vee\left(S_{n}(f)-\lambda e\right)^{+}\right.}\right)_{n \in \mathbb{N}}$ for fixed $\lambda>0$. Next, we will consider a sequence of stopping times and it would be suitable to introduce some additional notations:

$$
P_{n, k}=P_{\left(\sup _{i \leq n}\left(\left|f_{i}\right|-k e\right)^{+} \vee\left(S_{n}(f)-k e\right)^{+}\right.} \quad \text { and } \quad P(k)=\left(P_{n, k}\right)_{n \geq 1} .
$$

Theorem 3 will be applied to the sequence $\left(Q_{k}^{d}\right)$ where $Q_{k}$ is the band projections defined by

$$
Q_{k}=P_{\left(f^{*}-k e\right)^{+} \vee(S(f)-k e)^{+}}=\sup _{n} P_{n, k} .
$$

Thanks to Lemma 12 and Austin's Theorem for vector lattices [15, Theorem 4.1] we deduce that $S(f)$ and $f^{*}=\sup \left|f_{i}\right|$ belongs to $E^{u}$. It is easily seen that $Q_{k}^{d} g_{n}^{P(k)}=Q_{k}^{d} g_{n}$ for all integers $k$ and $n$. Thus, the sequence $\left(Q_{k}^{d} g_{n}\right)_{n \geq 1}$ is order convergent for every $k$. Moreover, it follows from Theorem 10 that $Q_{k}^{d} \uparrow I$. The proof is now completed by invoking Theorem 3 .

\section{References}

[1] Aliprantis, C., Burkinshaw, O.: Positive Operators. Springer, Dordrecht (2006)

[2] Azouzi, Y.: Completeness for vector lattices, J. Math. Anal. Appl. 472. 216-230 (2019) 
[3] Azouzi, Y., Ramdane, K.: Burkholder Inequalities in vector lattices, Indag. Math. (N.S.) 28(5), 1076-1094. (2017)

[4] Azouzi, Y., Trabelsi, M.: $L^{p}$-spaces with respect to conditional expectation on vector lattices, J. Math. Anal. Appl. 447. 798-816 (2017)

[5] Burkholder, D. L.: Martingale transforms, Ann. Math. Statist., 37. 1494-1504 (1966).

[6] Buskes, G., van Rooij, A.: Almost $f$-algebras: Commutativity and the Cauchy-Schwarz Inequality Positivity 4. 227-231 (2000).

[7] Chen, L. T. H.: Martingale convergence via the square function, Proc Amer. Soc. 83, 125-127 (1981).

[8] Chow, Y. S., Teicher, H.: Probability Theory, Springer, New York, 1988.

[9] Donner, K.: Extension of Positive Operators and Korovkin Theorems, Springer (1982)

[10] Gao, N., Troitsky, V. G., Xanthos, F.: Uo-convergence and its applications to Ces'aro means in Banach lattices, Israel J. Math, 220, 649-689 (2017)

[11] Grobler, J. J.: Continuous stochastic processes in vector lattices: the Doob-Meyer decomposition, Positivity, 14, 731-751 (2010)

[12] Grobler, J. J.: Jensen's and martingale inequalities in vector lattices, Indag. Math., 25, 275-295 (2014)

[13] Grobler, J. J. and Labuschagne, C. A.: The Itô integral for Brownian motion in vector lattices: Part 1., J. Math. Anal. Appl., 423, 797-819. (2015)

[14] Grobler, J. J. and Labuschagne, C. A.: The Itô integral for Brownian motion in vector lattices: Part 2., J. Math. Anal. Appl., 423, 820-833 (2015)

[15] Grobler, J. J. Labuschagne, C. A., and Marraffa, V.: Quadratic variation of martingales in vector lattices, J. Math. Anal. Appl., 410, 418-426 (2014) 
[16] Grobler, J. J., Schwanke, C. M.: Stopped processes and Doob's optional sampling theorem arXiv:2007.05171 [math.FA].

[17] Huijsmans, C.B., de Pagter,B.: Ideal Theory in f-Algebras, Trans. Amer. Math. Soc., 269, 225-245 (1982)

[18] Kaplan, S.: On unbounded order convergence, Real Anal. Exchange 23, 175-184. (1997/98)

[19] Kuo, W. C., Labuschagne, C. A. and Watson, B. A.: Conditional expectations on vector lattices, J. Math. Anal. Appl., 303, 509-521 (2005)

[20] Kuo, W. C., Labuschagne, C. A. and Watson, B. A.: Discrete-time stochastic processes on vector lattices, Indag. Math., 15, 435-451 (2004)

[21] Kuo, W. C., Labuschagne, C. A., Watson, B. A.: Convergence of vector lattice martingales. Indag. Mathem., 17, 271-283 (2006)

[22] Hui Li, Zili Chen,: Some loose ends on unbounded order convergence, Positivity 22(1), 83-90 (2018)

[23] Williams, D.: Probability with martingales, Cambridge University Press (1991)

[24] Zaanen, A. C.: Introduction to Operator Theory in vector lattices, Springer-Verlag, 1997. 